\title{
Isolation of the Bacteriophage DinoHI from Dichelobacter nodosus and its Interactions with other Integrated Genetic Elements
}

\author{
Brian F. Cheetham*,1, Dane Parker ${ }^{2}$, Garry A. Bloomfield ${ }^{1}$, Bruce E. Shaw ${ }^{1}$, Megan Sutherland ${ }^{1}$, \\ Jessica A. Hyman ${ }^{1}$, Jenifer Druitt ${ }^{1}$, Ruth M. Kennan ${ }^{2}$, Julian I. Rood ${ }^{2}$ and Margaret E. Katz ${ }^{1}$ \\ ${ }^{1}$ Molecular and Cellular Biology, University of New England, Armidale, NSW, 2351, Australia \\ ${ }^{2}$ Australian Research Council Centre of Excellence in Structural and Functional Microbial Genomics and Victorian \\ Bioinformatics Consortium, Department of Microbiology, Monash University, Clayton, Vic., 3800, Australia
}

\begin{abstract}
The Gram-negative anaerobic pathogen Dichelobacter nodosus carries several genetic elements that integrate into the chromosome. These include the int $A$, int $B$, int $C$ and int $D$ elements, which integrate adjacent to $\operatorname{csr} A$ and $p n p A$, two putative global regulators of virulence and the virulence-related locus, $v r l$, which integrates into $s s r A$. Treatment of $D$. nodosus strains with ultraviolet light resulted in the isolation of DinoHI, a member of the Siphoviridae and the first bacteriophage to be identified in D. nodosus. Part of the DinoHI genome containing the packaging site is found in all D. nodosus strains tested and is located at the end of the $v r l$, suggesting a role for DinoHI in the transfer of the $v r l$ by transduction. Like the intB element, the DinoHI genome contains a copy of regA which has similarity to the repressors of lambdoid bacteriophages, suggesting that the maintenance of DinoHI and the int $B$ element may be co-ordinately controlled.
\end{abstract}

\section{INTRODUCTION}

The Gram-negative anaerobic bacterium Dichelobacter nodosus is the principal causative agent of ovine footrot $[1,2]$. D. nodosus strains are classified as virulent, intermediate or benign depending on the severity of the disease which they cause in sheep. Comparative analysis of DNA from virulent and benign strains has led to the identification of a series of genetic elements which integrate into the $D$. nodosus chromosome. These include the int A [3-7], intB [8], int $C$ [8] and intD elements [9], Tanjung, L. and Cheetham, B.F., unpublished, each of which contains an integrase gene. A fifth element, the virulence-related locus, $v r l[6,10,11]$, lacks an integrase gene. The intA element and the $v r l$ locus are found in almost all virulent strains [12,13] and are absent from the majority of benign strains.

We have proposed a model for the modulation of virulence by integration of the int $A$, int $B$, int $C$ and int $D$ elements into two different tRNA-ser genes next to $\operatorname{csr} A$ (formerly $g l p A$ ) and $p n p A$, which encode putative global regulators of virulence [14]. CsrA and the closely related protein RsmA are virulence repressors in Salmonella enterica [15], Helicobacter pylori [16], Legionella pneumophila [17] and Erwinia carotovora [18]. The pnpA product, polynucleotide phosphorylase, is a global regulator of virulence in $S$. enterica [19]. The $v r l$ element integrates into ssrA [10], which encodes a tmRNA molecule that is involved in the removal of ribosomes stalled on mRNA molecules and in the subsequent proteolytic degradation of the resultant peptides [20]. In $S$. enterica, mutations in ssrA reduce the expression of genes from the virulence plasmid [21].

These integrated elements may have evolved from integrative plasmids or bacteriophages. To determine whether

*Address correspondence to this author at the Molecular and Cellular Biology, University of New England, Armidale, NSW, 2351, Australia; Tel: 61 26773 3394; Fax: 6126773 3267; E-mail: bcheetha@une.edu.au these elements behave as prophages, we treated several $D$. nodosus strains with agents known to induce prophages, and isolated a bacteriophage, DinoHI, from one strain. Cloning and analysis of the DinoHI genome showed that it was distinct from all previously-characterised integrated genetic elements of D. nodosus, and suggested that it may have a role in the transfer of the $v r l$ locus by transduction.

\section{MATERIALS AND METHODOLOGY}

\section{General Methods}

Methods for the growth of D. nodosus, preparation of genomic DNA, cloning and analysis of DNA, Southern blotting, DNA sequencing and DNA sequence analysis, together with the source of $D$. nodosus strains have been reported previously $[7,8,14]$. The nucleotide sequence of the NruIHindIII fragment containing the int $P$ gene has been given the GenBank accession no. EU048235, and the junction fragment spanning attL in strain $\mathrm{H} 1215$ has been given the GenBank accession no. EU048236.

\section{Bacteriophage Induction - Mitomycin C}

D. nodosus strains were inoculated into $10 \mathrm{ml}$ of Eugonbroth (Becton-Dickinson) and grown under anaerobic conditions at $37^{\circ} \mathrm{C}$ for $48 \mathrm{~h}$. Mitomycin $\mathrm{C}$ was added at varying concentrations from 0.2 to $2 \mu \mathrm{g} / \mathrm{ml}$ and the cultures incubated for a further $24 \mathrm{~h}$. The bacterial cells were removed by centrifugation at $3000 \times \mathrm{g}$ for $15 \mathrm{~min}$ and the supernatant was filtered through a $0.45 \mu \mathrm{m} \mathrm{HV}$ filter (Millipore). Phage particles were collected by centrifugation at 32,000 x $g$ for 2 $\mathrm{h}$ at $4{ }^{\circ} \mathrm{C}$ and resuspended in $100 \mu \mathrm{l}$ of $\mathrm{SM}$ [100 mM NaCl, 8

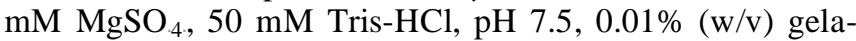
tin].

\section{Bacteriophage Induction - Ultraviolet Light}

D. nodosus strains were inoculated into $10 \mathrm{ml}$ of Eugonbroth and grown for $48 \mathrm{~h}$. Cells were collected by centrifugation at 3,000 x $g$ for $5 \mathrm{~min}$, resuspended in $300 \mu \mathrm{l}$ of $10 \mathrm{mM}$ 


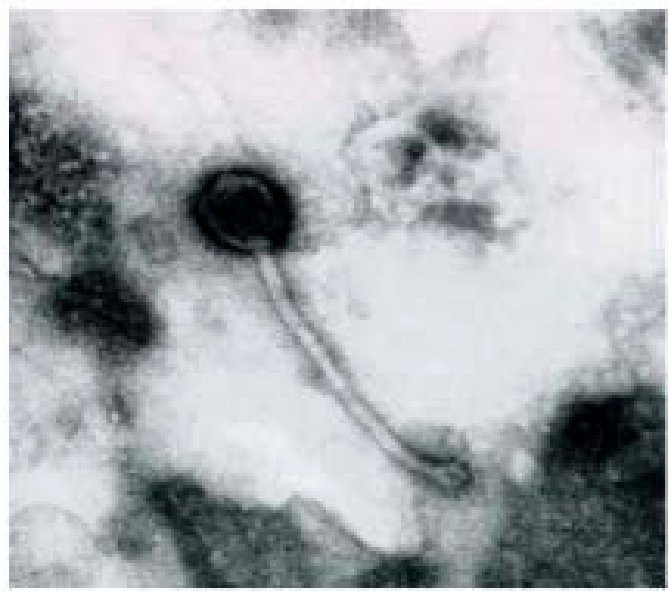

Fig. (1). Electron micrograph of DinoHI stained with uranyl acetate. Scale bar $=50 \mathrm{~nm}$.

$\mathrm{MgSO}_{4}$. [22] and then the suspension was placed in a petri dish $35 \mathrm{~cm}$ from a 15 Watt ultraviolet germicidal lamp (intensity approximately 1000 lux) and irradiated for 10 to $20 \mathrm{~s}$. The irradiated suspension was inoculated into $10 \mathrm{ml}$ of Eugonbroth and incubated at $37^{\circ} \mathrm{C}$ for $24 \mathrm{~h}$ in the dark. Bacteriophage particles were collected by centrifugation as described above.

\section{Electron Microscopy}

Phage preparations were diluted 1:3 in Hungate's salts solution, then an $8 \mu \mathrm{l}$ drop was allowed to settle for $10 \mathrm{~min}$ on $0.25 \%(\mathrm{v} / \mathrm{v})$ formvar-coated copper mesh grid and the access liquid removed [23]. The samples were stained with either 2\% (w/v) potassium phosphotungstic acid $(\mathrm{pH} 7.0)$ for 20 to $25 \mathrm{~s}$ or $4 \%$ (w/v) uranyl acetate $(\mathrm{pH} 4.0)$ for 5 to $10 \mathrm{~s}$ [24]. The prepared grids were air dried and examined using a Phillips 300 transmission electron microscope. Phage particles were photographed at a magnification of $100,000 \mathrm{x}$.

\section{RESULTS AND DISCUSSION}

\section{Induction of a Bacteriophage from $D$. nodosus Strain H1215}

Liquid cultures of D. nodosus strains A198, AC3577, C305, G1220, H1204 and H1215 were treated with mitomycin $\mathrm{C}$ or ultraviolet light, two commonly used agents for the induction of prophage excision [25]. These strains cover a range of $D$. nodosus serogroups and virulence classifications. After growth to stationary phase, bacterial cells were removed by centrifugation, the supernatant was subjected to high speed centrifugation to sediment bacteriophage particles, and any potential nucleic acid was extracted from the pellet. Agarose gel electrophoresis showed a band of greater than $23 \mathrm{~kb}$ in extracts from only one strain, H1215, and only after treatment with ultraviolet light. This result suggested that a bacteriophage had been induced. No such band was seen in uninduced cultures of strain H1215.

\section{Characterisation of Bacteriophage DinoHI by Electron Microscopy}

Bacteriophage preparations for electron microscopy were negatively stained with either phosphotungstic acid or uranyl acetate and observed at a $100,000 \times$ magnification. Phage particles (Fig. 1) were of uniform size and comprised an icosahedral head, $57 \mathrm{~nm}$ apical diameter, and a tail, $10 \mathrm{~nm} \mathrm{x}$

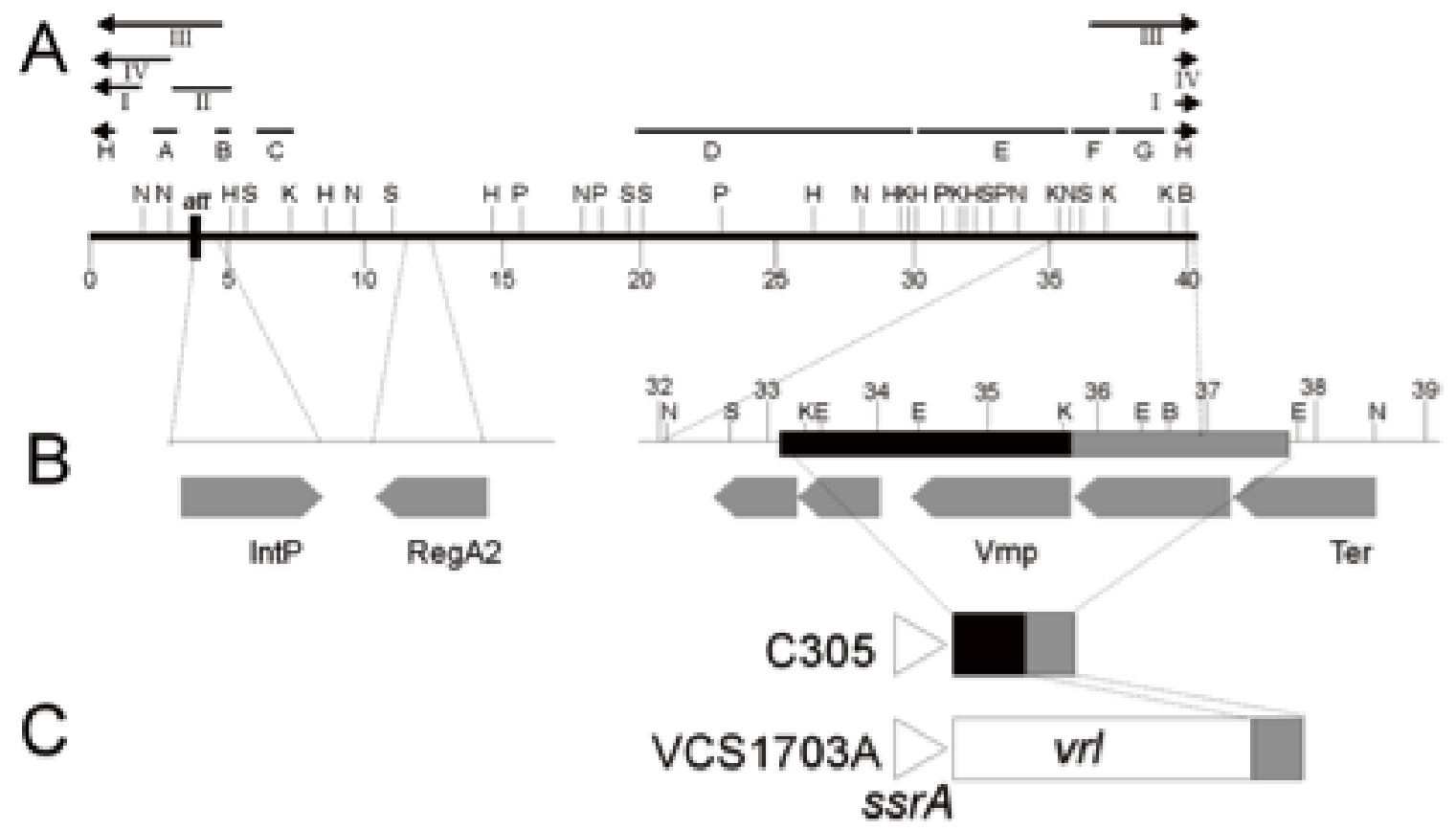

Fig. (2). A. Restriction enzyme map of the linear form of the DinoHI genome. Restriction enzyme sites shown are BamHI (B), EcoRI (E), $\operatorname{HindIII}(\mathrm{H}), \operatorname{KpnI}(\mathrm{K}), \operatorname{NruI}(\mathrm{N}), \operatorname{Pst} \mathrm{I}(\mathrm{P})$ and $\operatorname{SacI}(\mathrm{S})$. Distances in kb from the left hand linear end are shown on the scale line. The locations of probes A-H and fragments I-IV are shown. B. Expanded view of DinoHI map. Distances on the scale line are measured from the attL site. The integrase gene (IntP), regA2 (RegA2), putative virion morphogeneis protein (Vmp) and large terminase subunit (Ter) genes are indicated by grey arrows. C. Arrangement of sequences from the DinoHI genome repeated in strains VCS1703A and C305. The diagram is not to scale. 
$203 \mathrm{~nm}$, with a claw-like base plate, $13 \mathrm{~nm} \times 15 \mathrm{~nm}$. The dimensions of the tail indicate that it is non-contractile [26]. The phage head appears pentagonal, which is a distinguishing feature of icosahedral phage heads [27]. This is the first bacteriophage which has been isolated from D. nodosus, and it has been named DinoHI, from the host bacterial genus, species and strain [27].

\section{Characterisation of Phage Nucleic Acid and Classifica- tion of DinoHI}

The nucleic acid isolated from the bacteriophage preparation was sensitive to DNaseI and was digested by restriction enzymes, indicating that the genome was double-stranded DNA. Pulsed field gel electrophoresis showed that the genome consisted of a single linear molecule of approximately $43 \mathrm{~kb}$ (data not shown). From the morphology and genome structure, DinoHI belongs to the virus family Siphoviridae [26].

\section{Restriction Enzyme Map of DinoHI}

Restriction enzyme analysis of DinoHI-derived DNA and of fragments from the DinoHI genome cloned into pUC18 was used to construct a map (Fig. 2A). The sizes of the restriction fragments obtained by digestion of DinoHI DNA were consistent with a circular map, indicating that the linear ends of the DNA are held together tightly and are not easily separated by routine agarose gel electrophoresis (data not shown). Tight binding of fragments containing the linear ends of phage CNRZ1205 from Streptococcus thermophilus has been observed previously [28]. Some bands from restriction enzyme fragments spanning the unique BamHI site in the DinoHI genome were present in submolar concentrations. This indicates that the linear ends are located close to this site, and there is a mixture of dissociated fragments and fragments in which the ends are still joined.

\section{Identification of the DinoHI Integrase Gene}

The int $A$, intB, int $C$ and int $D$ elements of $D$. nodosus integrate such that the integrase gene is located approximately $200 \mathrm{nt}$ from the att site [4,8]. A similar arrangement is seen for bacteriophages of the P4 family [29]. The D. nodosus integrases are related to the $\mathrm{P} 4$ integrase $[4,8]$. If the same were true for DinoHI, then the integrase gene would be found on restriction fragments which span the integration site. These fragments would be expected to be a different size in the genome of DinoHI compared to the genome of $D$. nodosus strain H1215, which contains the integrated form of the bacteriophage. Comparative Southern blot analysis of DNA from DinoHI and strain H1215 using a series of probes spanning the DinoHI genome (probes A-H in Fig. 2A) identified several restriction fragments whose sizes differed in the genomes of DinoHI and strain H1215. Fig. (3) shows a sample of this data, where a $1.0 \mathrm{~kb} \mathrm{NruI}$ fragment hybridises to a fragment of the same size in both DinoHI and H1215 (panel A), while the adjacent $2.2 \mathrm{~kb} \mathrm{NruI-HindIII} \mathrm{fragment}$ hybridises to a $2.2 \mathrm{~kb}$ fragment in DinoHI, but to two fragments of $1.8 \mathrm{~kb}$ and $1.5 \mathrm{~kb}$ in $\mathrm{H} 1215$ (panel B). Thus, the integration site was localised to this $2.2 \mathrm{~kb} \mathrm{NruI} / H$ HindIII fragment (fragment II in Fig. 2A), whose sequence was determined (GenBank accession no. EU048235). This fragment contained an ORF of $1197 \mathrm{nt}$, whose predicted protein product, designated IntP, has $37 \%$ amino acid identity with the integrase of a symbiosis island from the nitrogen-fixing bacterium Mesorhizobium loti [30]. In addition, IntP has His at position 345, Arg at 348 and Tyr at 381, which align with residues conserved between a number of bacteriophage integrases [31]. IntP has approximately $32 \%$ amino acid identity with the integrases identified in the $D$. nodosus int $A$, int $B$, int $C$ and int $D$ elements.

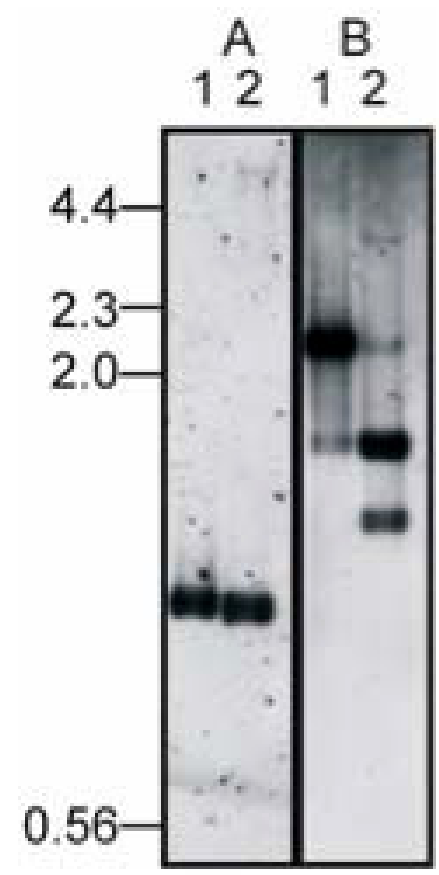

Fig. (3). Identification of integration site. Genomic DNA from DinoHI (lane 1) and D. nodosus strain H1215 (lane 2) was digested with NruI (panel A) or NruI and HindIII (panel B) and probed in panel A with a $1.0 \mathrm{~kb} \mathrm{NruI}$ fragment from DinoHI (probe A, Fig. 1A) or in panel B with a $2.2 \mathrm{~kb} \mathrm{NruI-HindIII} \mathrm{from} \mathrm{DinoHI} \mathrm{(frag-}$ ment II, Fig. 2A). Sizes in kb of molecular weight markers are shown on the left hand side.

\section{Complete Sequence of Bacteriophage DinoHI}

The sequences of both ends of twenty one subclones from the DinoHI genome were determined. While the characterisation of DinoHI was in progress, the complete sequence of the genome of $D$. nodosus strain VCS1703A was determined [32]. Blast searches of the D. nodosus genome sequence using the sequences from DinoHI showed that strain VCS1703A contained an integrated copy of the DinoHI genome. Sequences from DinoHI were almost identical to the corresponding sequences in the genome of strain VCS1703A, as were the restriction maps, with a small number of restriction site polymorphisms.

Analysis of the DinoHI genome revealed the presence of 47 ORFs (Table 1). Of these genes and their gene products, 22 had no homologues in the databases, 14 had homologues of unknown function, 7 had homologues from other bacteriophages and 4 had homologues with roles in DNA replication.

\section{Southern Hybridisation Analysis of other Strains of $D$. Nodosus}

Southern blots were carried out on genomic DNA from eighteen D. nodosus strains using probes A-H (Fig. 2) span- 
Table 1. Putative ORFs from DinoHI. $(c) h p=($ Conserved $)$ Hypothetical Protein. Coordinates are Taken from GenBank Accession no. CP000513

\begin{tabular}{|c|c|c|}
\hline Start & Stop & Product/gene \\
\hline 788865 & 789692 & chp - membrane \\
\hline 789685 & 789960 & hp \\
\hline 789863 & 790168 & hp \\
\hline 790165 & 790956 & hp \\
\hline 790934 & 792208 & phage terminase, large subunit \\
\hline 792205 & 793662 & chp, pseudo \\
\hline 793659 & 795158 & phage virion morphogenesis-like protein \\
\hline 795669 & 796067 & hp \\
\hline 796078 & 796899 & hp \\
\hline 796902 & 797888 & chp \\
\hline 797910 & 798377 & chp \\
\hline 798374 & 798799 & phage virion morphogenesis-like protein \\
\hline 798780 & 799247 & hp \\
\hline 799244 & 800011 & chp \\
\hline 800014 & 800241 & hp \\
\hline 800249 & 800458 & hp \\
\hline 800495 & 804466 & phage tail tape measure family protein \\
\hline 804463 & 804867 & chp \\
\hline 804876 & 808505 & chp \\
\hline 808507 & 809373 & $\operatorname{chp}$ \\
\hline 809285 & 809710 & hp \\
\hline 809707 & 811383 & $\operatorname{chp}$ \\
\hline 811392 & 811619 & hp \\
\hline 812618 & 812055 & hp \\
\hline 814279 & 812666 & helicase family protein \\
\hline 815422 & 814328 & hp \\
\hline 816242 & 815574 & hp \\
\hline 816617 & 816363 & hp \\
\hline 817090 & 816635 & single-strand binding protein/ss $b$ \\
\hline 817960 & 817103 & $\operatorname{chp}$ \\
\hline 818562 & 817957 & bacteriophage recombination function protein \\
\hline 818920 & 818588 & hp \\
\hline 819207 & 818920 & hp \\
\hline 820748 & 819315 & chp \\
\hline 821794 & 820877 & regulatory protein/regA2 \\
\hline 822196 & 822459 & $\mathrm{hp}$ \\
\hline
\end{tabular}

(Table 1). Contd.....

\begin{tabular}{|c|c|c|}
\hline Start & Stop & Product/gene \\
\hline \hline 822460 & 823347 & chp \\
\hline 823352 & 824116 & DNA replication protein/dnaC \\
\hline 824871 & 825815 & chp \\
\hline 824363 & 825878 & hp \\
\hline 825812 & 826231 & hp \\
\hline 826234 & 826611 & endodeoxyribonuclease RusA family \\
\hline 826605 & 826961 & hp \\
\hline 826961 & 827212 & chp \\
\hline 827196 & 827603 & hp \\
\hline
\end{tabular}

ning most of the DinoHI genome (Table 2). Genomic DNA from six of these strains hybridised to all probes from the DinoHI genome, indicating that these strains contained a complete integrated copy of the bacteriophage. Bacteriophages were successfully induced from all of these strains after treatment with ultraviolet light. Of these strains, five are virulent and one is benign. Although almost all of the strains tested which contained DinoHI were virulent, it is unlikely that DinoHI carries genes with an essential role in virulence, as the bacteriophage is not found in strain A198, the reference virulent strain, and is also not present in the virulent strain G1220.

Genomic DNA from strain 3526 hybridised to most of the probes tested, but did not hybridise to probe $\mathrm{B}$, which contains the integrase gene, int $P$, and also failed to hybridise to probe $\mathrm{D}$, which spans $10 \mathrm{~kb}$ of the DinoHI genome. Bacteriophage particles were successfully induced from this strain, and the genome size was similar to that of DinoHI. The bacteriophage from strain 3526 appears to be related to DinoHI, but has a different integrase and other sequences which differ from DinoHI.

Genomic DNA from all strains hybridised to probes $G$ and H, and DNA from strains C305, G1220 and H1204, which did not hybridise to most of the DinoHI probes, also hybridised to probe F. This result suggests that part of the DinoHI genome is present in all strains, and the size of this region varies between strains. Strains which contained the complete DinoHI genome had two copies of sequences detected by probes $\mathrm{G}$ and $\mathrm{H}$, and strains which did not contain DinoHI had only one copy (data not shown). Probe G contains a phage virion morphogenesis-like protein gene, while Probe $\mathrm{H}$ contains part of the putative phage terminase gene (Table 1).

\section{Identification of a New Integrated Genetic Element in Strain H1215}

A $6 \mathrm{~kb}$ SacI restriction fragment that contained the DinoHI integrase gene was cloned from genomic DNA of strain H1215 to construct pBSD32.4. When used to probe genomic DNA from strain $\mathrm{H} 1215$ digested with SacI, pBSD32.4 detected a single band of $6 \mathrm{~kb}$. However, when used to probe DNA from DinoHI digested with $S a c I$, it de- 
Table 2. Hybridisation of Genomic DNA from D. Nodosus Strains to Probes from the DinoHI Genome (Fig. 2A). + Indicates Hybidisation, - Indicates no Hybridisation

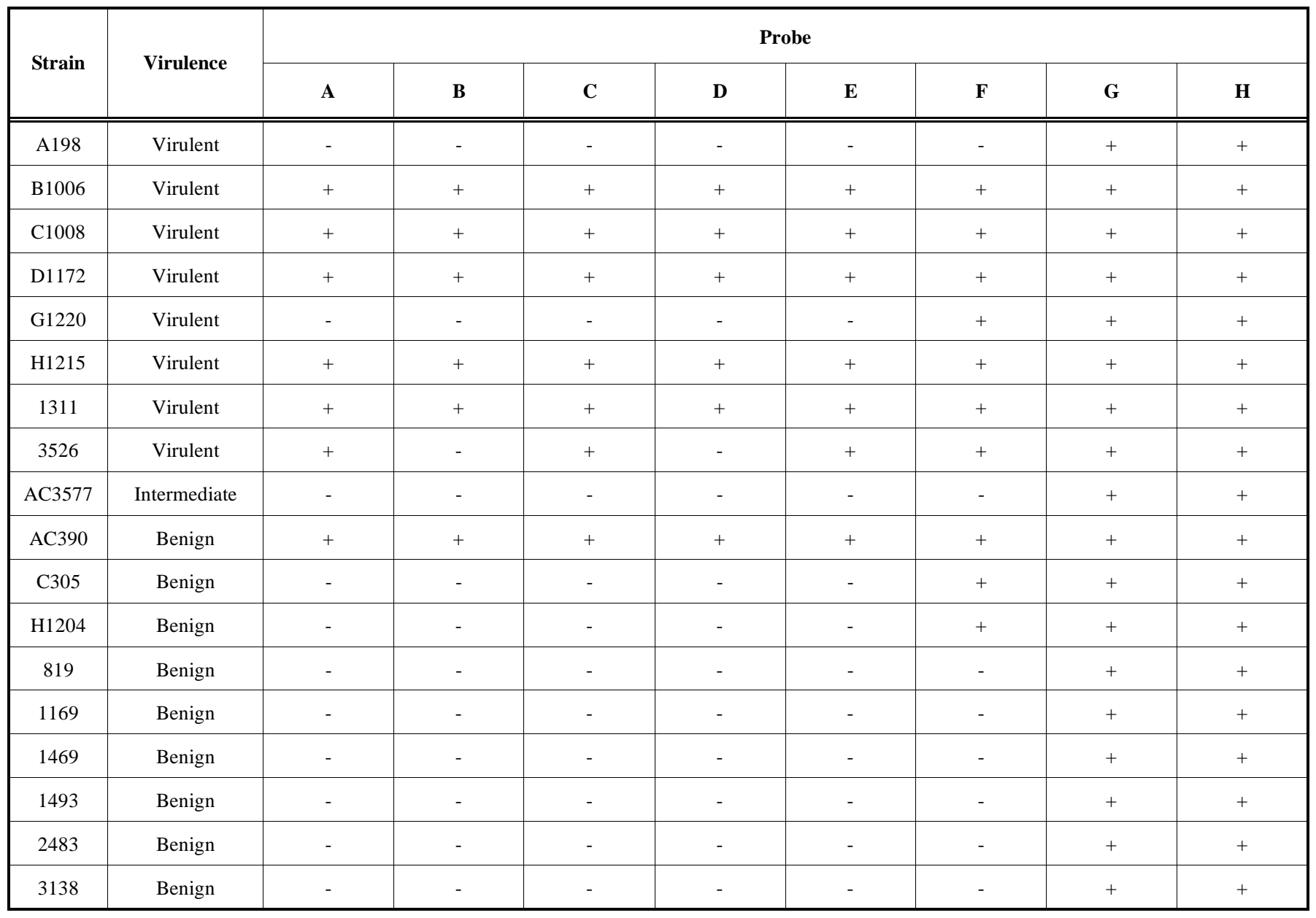

tected a band of $11 \mathrm{~kb}$ (fragment III, Fig. 2A). These results indicated that pBSD32.4 spanned the site of integration, as it contained the DinoHI integrase together with chromosomal sequences from strain H1215 which are not present in the DinoHI genome.

The sequence of part of pBSD32.4 was determined (GenBank accession no. EU048236). The DNA sequences starting $240 \mathrm{nt}$ upstream from the integrase genes in pBSD32.4 (which is the integrated form in strain H1215), VCS1703A [32] and the DinoHI genome were aligned (Fig. 4A). All three sequences differed for the first $51 \mathrm{nt}$, and were identical after this point, suggesting that the att site is found $189 \mathrm{nt}$ upstream of the integrase gene. This is the approximate position of the att sites of the int $A$, int $B$ and int $C$ elements of D. nodosus $[4,8]$ and of bacteriophages such as $\mathrm{P} 4$ $[33,34]$. The att site for DinoHI was defined as the $20 \mathrm{nt}$ sequence TTTGTATGATGTGGGCATCA from DinoHI (GenBank accession no EU048235, bold in Fig. 4) which shows $90 \%$ identity at the left junction (attL, upstream from int $P$ ) and $80 \%$ identity at the right junction (attR, Fig. 4B) with genomic DNA from strain VCS1703A. Thus, the integrated DinoHI genome spans positions 829484 to 789155 in D. nodosus strain VCS1703A (GenBank accession no. CP000513).
The differences between the sequences on the left side of attL (Fig. 4A) in strains H1215 and VCS1703A could be explained if DinoHI integrates at a different site in the genomes of these two strains. PCR primers were designed based on the genomic sequence of VCS1703A to amplify the fragments at the left and right junctions of the integrated bacteriophage (Fig. 5). Primers C and D spanning the right hand junction amplified a fragment of the same size in strains VCS1703A and H1215, while primers A and B spanning the left hand junction amplified a fragment in VCS1703A, but not H1215 (data not shown). Sequences from pBSD32.4 on the left side of the $a t t L$ were not detected in the VCS1703A genome by blast searches, so these sequences are absent from this strain. This may have arisen by the deletion of these sequences in strain VCS1703A. However, another possible explanation for these findings is that DinoHI is located at the same site in strains H1215 and VCS1703A, but another integrated element is present on the left hand side of DinoHI in strain H1215. The tandem integration of genetic elements has been observed previously in D. nodosus [8]. In addition, the sequences from pBSD32.4 which are absent from strain VCS1703A contain a type I restriction-modification system (Cheetham, B.F., Hyman, J. and Shaw, B.E., unpublished). Restriction-modification systems are often associated with mobile genetic elements [35]. 

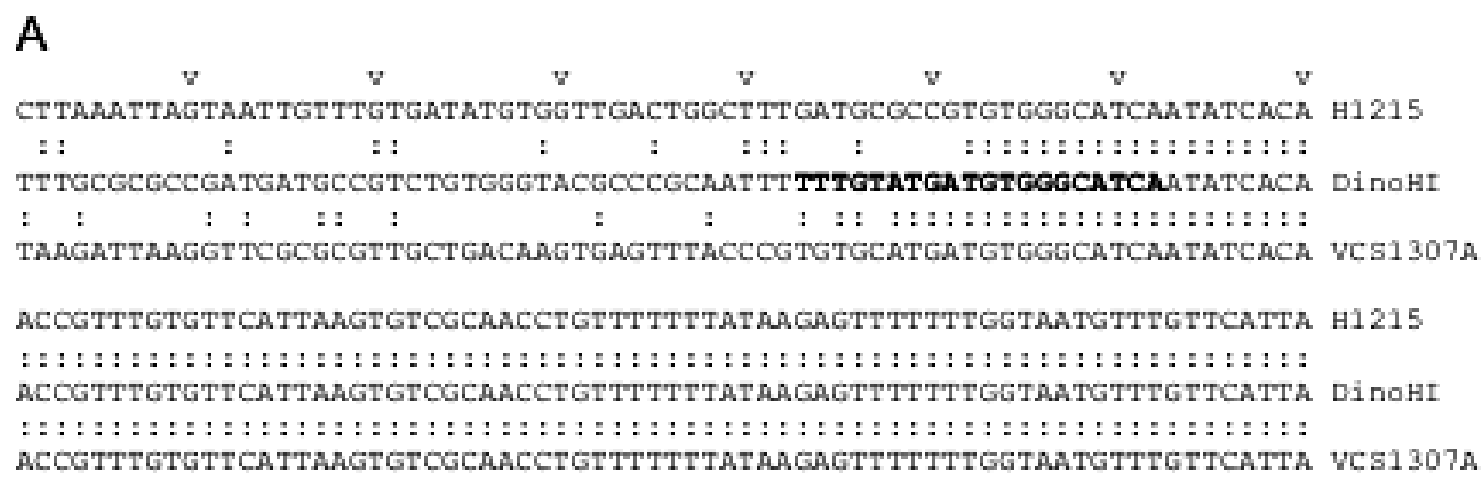

B TGACGCGCGTTGTCCAGCCGCGCTTAAAGGCATGCCAATTTTAAGACGTTTGAGGAATGCCATGCGTTC DI NOHI : : : : : : : : : : : : : : : : : : : : : : : : : : : : : : : : : : : : : : : : : : : : : : : : : : : : : : : : : : : : : : : : : : : : : : TGACGCGCGTTGTCCAGCCGCGCTTAAAGGCATGCCAATTTTTAAGACGTTTGAGGAATGCCATGCGTTC VCS1703A

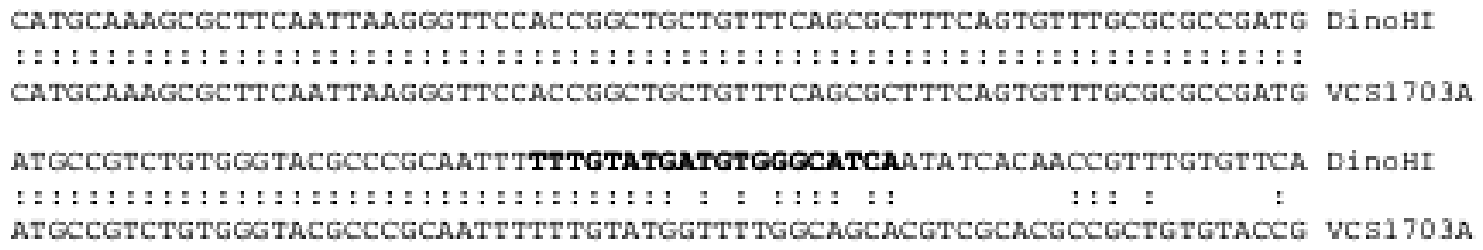

Fig. (4). A. Position of attL. Alignment of sequences beginning $240 \mathrm{nt}$ upstream from the start codon of the intP genes of $D$. nodosus strain H1215 (nt 340-549 of GenBank accession no. EU048236) and and VCS1703A (complement of 829317 to 829526 of GenBank accession no. CP000513) with DinoHI (nt 442 to 651 of GenBank accession no. EU048235). Every 10P ${ }^{\text {thP }}$ nucleotide is marked by v. Identical nucleotides are joined by colons. B. Positon of attR. Alignment of nt 316 to 525 of DinoHI (GenBank accession no. EU048235) with the complement of nt 789133 to 789342 of the VCS1703A genome (GenBank accession no. CP000513).

The putative new integrated element in strain H1215 adjacent to DinoHI has been designated "element X" (Fig. 5A).

The int $A$, intB, int $C$ and intD elements of $D$. nodosus integrate into tRNA genes and the $v r l$ integrates into $s s r A$. No genes encoding small RNA molecules were found to the left of the DinoHI attL site in VCS1703A (Fig. 4A). However, integration of DinoHI into strain VCS1703A creates an ORF that potentially encodes a 275 aa protein (DN_0739, Table 1) that has amino acid similarity to several secretion activator proteins [36], of which two are located on phage genomes [37,38]. The N-terminal 97 aas of this ORF are encoded to the right of $a t t R$, and the remaining 178 are encoded by the DinoHI genome. Homology is seen over sequences encoded both by the DinoHI genome and the chromosome.

The plasmid pBSD32.4 (Fig. 5B), containing sequences from both DinoHI and the adjacent region (the putative element X) in strain H1215 was used to probe genomic DNA digested with HindIII from sixteen strains of D. nodosus. Five different patterns were seen on this Southern blot. Genomic DNA from strains A198, C305, AC3577, 819, 1169, 2483, 1493, 3138 and 1469 did not hybridise to pBSD32.4. These strains do not contain DinoHI (Table 1), and this result suggests that they do not contain element $\mathrm{X}$. There were two bands of $15 \mathrm{~kb}$ and $3.1 \mathrm{~kb}$ in DNA from strains 1311 , B1006, D1172 and C1008. These strains contain DinoHI (Table 2), but do not contain element X. DNA from strain H1215, which contains both DinoHI and element $X$, gave bands of 12.8, 3.1, 3.0 and $1.8 \mathrm{~kb}$. Bands of 12.8, 3.0 and 1.8 $\mathrm{kb}$ were detected in DNA from strains G1220 and H1204, indicating that these strains contain element $\mathrm{X}$, but not a complete copy of DinoHI. Finally, DNA from strain JIR1212 gave bands of $15.0,12.8,3.1,3.0$ and $1.8 \mathrm{~kb}$. This strain appears to contain both DinoHI and element $X$, but the arrangement differs from strain H1215. One possible explanation is that there is a copy of DinoHI at each end of element $X$. Thus, element $X$ was present in only four out of sixteen strains tested. Of these, three were virulent and one was benign.

\section{A Copy of $\operatorname{reg} A$ in the DinoHI Genome}

The int $B$ element of $D$. nodosus contains a gene, regA, which is related to several regulatory proteins, including the cI repressor from lambdoid bacteriophages [8]. Blast search analysis of the DinoHI genome showed that it also contained a copy of regA, designated regA2 (Fig. 2A). Comparison of the sequences from the intB element of $D$. nodosus strain A198 and the DinoHI genome revealed a 701 nt sequence with $97 \%$ identity. This identity begins immediately after the 


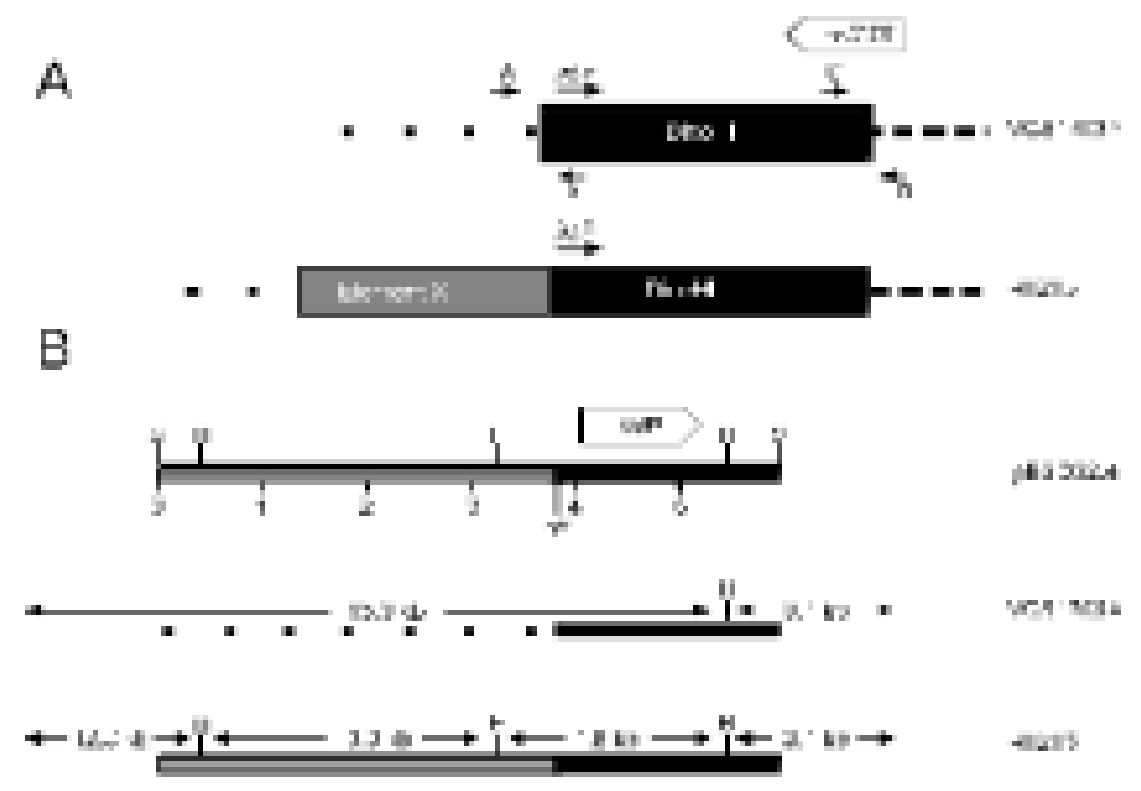

Fig. (5). A. The predicted arrangement of DinoHI and Element X in the genomes of strains VCS1703A and H1215. The positions of primers A, B, C and D are shown by small arrows. The diagram is not drawn to scale. B. Restriction map of clone pBSD32.4 and the genomic arrangement in strains VCS1703A and H1215. Restriction sites shown are HindIII (H) and SacI (S). The scale line gives distance in kb. Sizes of restriction fragments in strains VCS1703A and H1215 are indicated.

start codon of the int $B$ element regA, and ends $25 \mathrm{nt}$ after the stop codon. The predicted aa sequence for the two products is $100 \%$ identical for 231 of the 232 amino acids of the intB regA. However, regA2 lacks the start codon found in $\operatorname{reg} A$. Instead, there are two potential start codons upstream in the same reading frame, which would result in an additional 17 or 144 aa at the N-terminus. The predicted length of the intBencoded RegA protein is very close to that of closely-related proteins identified through blast searches, typically 230-231 aa.

The high level of nt identity between $\operatorname{reg} A$ and $\operatorname{reg} A 2$ suggests a recent duplication, or a selective mechanism for the maintenance of the DNA sequence. Analysis of the junction regions did not reveal any special features such as repeated sequences. By contrast, the intA element contains repeats of a $102 \mathrm{nt}$ and a $103 \mathrm{nt}$ repeat and some duplicated sequences from the intA element are bounded by these repeats [9].

Expression of $\operatorname{reg} A$ and $\operatorname{reg} A 2$ would lead to the production of two proteins which are almost identical, except for an additional sequence at the $\mathrm{N}$-terminus in RegA2. These proteins are highly related at the aa level to repressor proteins of lambdoid bacteriophages, which act to maintain these phages in the integrated state. It is possible that RegA maintains the int $B$ element in an integrated state, and RegA 2 maintains the DinoHI prophage. However, the high structural similarity could allow co-ordinate control of these two genetic elements, as each repressor may act on both integrated elements.

\section{Mapping of the Linear Ends of DinoHI}

Southern blots of DinoHI DNA digested with several restriction enzymes and probed with DNA from several regions of the DinoHI genome showed the presence of faint bands (data not shown), consistent with pieces containing the linear ends. In particular, DinoHI DNA digested with KpnI and EcoRV and probed with a $2.1 \mathrm{~kb} B a m \mathrm{HI} / N r u \mathrm{I}$ fragment (probe I, Fig. 2) detected a strong band of $4.3 \mathrm{~kb}$ (fragment IV, Fig. 2) and two faints bands of 3.0 and $1.3 \mathrm{~kb}$, localising the linear end to approximately $0.4 \mathrm{~kb}$ from the Bam HI site, about 37,000 nt from the att site (Fig. 2A). This site is immediately downstream from the terminase gene, and in several other lambdoid bacteriophages the linear ends are located close to the terminase gene $[28,39,40]$. At position 36952 in DinoHI, the sequence CGGGGCGGCG is found, which perfectly matches part of the bacteriophage lambda $\cos$ site [41]. An $11 \mathrm{nt}$ sequence is repeated at positions 36748 and 36897, resembling the arrangement of repeat sequences which direct the nicking of lambda DNA at the cos site [42]. Thus, the Southern blot evidence and sequence information support the location of the linear ends of DinoHI at this position.

\section{The DinoHI Packaging Site is Found at the End of the vrl Region}

Southern blot analysis (Table 1) showed that all strains hybridised to probes $\mathrm{G}$ and $\mathrm{H}$ (Fig. 2A), even if they did not contain the rest of the DinoHI genome, and some strains lacking DinoHI also hybridised to probe F. This was investigated by Blast searches using the DinoHI genome sequence, numbered from the beginning of the attachment site immediately upstream from intP. Nt 1-40318 of the DinoHI genome correspond to nt 829484-789167 of the D. nodosus strain VCS1703A genome. Nt 35837-37637 of the DinoHI genome were found to be present at the end of the $v r l$ in strain VCS1703A and A198 [10]. The linear ends of DinoHI have been mapped to within $100 \mathrm{bp}$ of position 37,000 (Fig. 2B), which is within this repeated fragment. The presence of the DinoHI packaging site at the end of the $v r l$ suggests that the 
$v r l$ region may be transferred between $D$. nodosus strains by transduction. Alternatively, the $v r l$ may be a prophage that has an identical packaging site to that of DinoHI.

Genomic DNA from strain C305, which lacks the vrl region, hybridises to probes $\mathrm{F}, \mathrm{G}$ and $\mathrm{H}$. A $3.5 \mathrm{~kb}$ fragment was amplified from strain C305 [10], using primers from within ssrA and within the region of DinoHI which is repeated at the end of the $v r l$ (Fig. 2C). Sequencing of the ends of this fragment indicated that the fragment contains nt 33053-35837 from DinoHI. Thus, there is a $4.6 \mathrm{~kb}$ segment of DinoHI, which includes the packaging site, next to $s r A$ in strain $\mathrm{C} 305$. This arrangement could have been generated by integration of DinoHI into ssrA, followed by deletion of most of the DinoHI genome. However, ssrA is not the integration site for DinoHI in either strain H1215 or VCS1703A. This segment of DinoHI contains a gene involved in virion morphogenesis and part of the terminase gene. The significance of this arrangement is not clear.

\section{CONCLUSIONS}

We have identified a new bacteriophage, DinoHI, with an integrase that is very similar to the integrases in the intA, int $B$, int $C$ and int $D$ elements. The discovery that the packaging site of DinoHI is located at the end of the $v r l$ region (Fig. 6) suggests that the $v r l$ may be transferred by transduction.

The finding that the DinoHI genome contains a copy of $\operatorname{reg} A$, a gene found on the int $B$ element and encoding a protein with similarity to the repressors of lambdoid phages, suggests that there may be coordinate regulation of the maintenance of the intB element and DinoHI. Similarly, the int $A$, int $C$ and intD elements all have genes designated vap $G$ and $v a p H$, (Fig. 6) whose arrangement resembles that of the immunity region of bacteriophage $\mathrm{P} 4$ [43]. In P4, this immunity region contributes to the maintenance of the bacteriophage in an integrated state, and prevents superinfection. Thus, the maintenance of the int $A$, int $C$ and int $D$ elements may be also co-ordinately regulated. Altogether, these results suggest that the integrated elements of D. nodosus are not independent, but instead are part of a complex regulatory system which controls the expression of virulence genes.

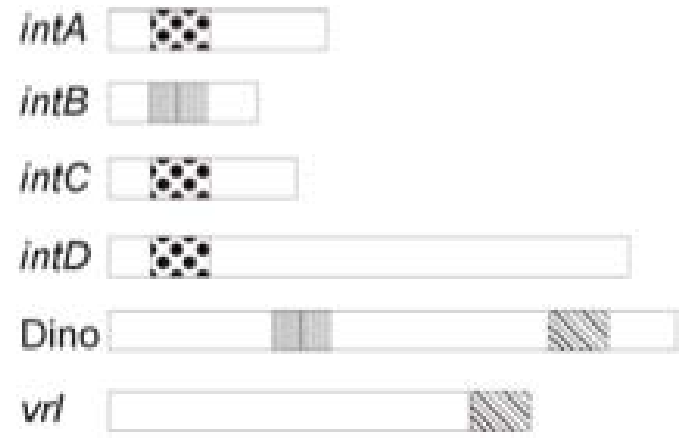

Fig. (6). Common regulatory sequences in the integrated elements of $D$. nodosus. The positions of vapH/vapG (dotted), regA (vertical bars) and the DinoHI packaging site $\left(45 \mathrm{P}^{\mathrm{oP}}\right.$ bars) in the int $A$, int $B$, int $C$ and intD elements, the $v r l$ and DinoHI are shown. The diagram is not drawn to scale.

\section{ACKNOWLEDGEMENTS}

The work carried out at the University of New England was supported by the Australian Research Council and the
University of New England. We thank Drs I Paulsen and G. Myers from TIGR for providing the D. nodosus VCS1703A sequence data prior to publication. Work at Monash University was supported by grants from the Australian Research Council.

\section{REFERENCES}

[1] Beveridge WIB. Footrot in sheep: a transmissible disease due to infection with Fusiformis nodosus. CSIRO Bull 1941; 140:1-40.

[2] Billington SJ, Johnston JL, Rood JI. Virulence regions and virulence factors of the ovine footrot pathogen, Dichelobacter nodosus. FEMS Microbiol Lett 1996; 145(2):147-156.

[3] Billington SJ, Sinistaj M, Cheetham BF, et al. Identification of a native Dichelobacter nodosus plasmid and implications for the evolution of the vap regions. Gene 1996; 172:111-116.

[4] Cheetham BF, Tattersall DB, Bloomfield GA, Rood JI, Katz ME. Identification of a gene encoding a bacteriophage-related integrase in a vap region of the Dichelobacter nodosus genome. Gene 1995; 162(1):53-58.

[5] Katz ME, Strugnell RA, Rood JI. Molecular characterization of a genomic region associated with virulence in Dichelobacter nodosus. Infect Immun 1992; 60:4586-4592.

[6] Katz ME, Howarth PM, Yong WK, Riffkin GG, Depiazzi LJ, Rood JI. Identification of three gene regions associated with virulence in Dichelobacter nodosus, the causative agent of ovine footrot. J Gen Microbiol 1991; 137:2117-2124.

[7] Katz ME, Wright CL, Gartside TS, et al. Genetic organization of the duplicated vap region of the Dichelobacter nodosus genome. J Bacteriol 1994; 176:2663-2669.

[8] Bloomfield GA, Whittle G, McDonagh MB, Katz ME, Cheetham BF. Analysis of sequences flanking the vap regions of Dichelobacter nodosus: evidence for multiple integration events, a killer system, and a new genetic element. Microbiology 1997; 143(Pt 2):553-562.

[9] Cheetham BF, Whittle G, Katz ME. Are the vap regions of the Dichelobacter nodosus genome pathogenicity islands? In: Kaper JB, Hacker J, editors. Pathogenicity Islands. Washington: ASM Press, 1999: 203-218.

[10] Haring V, Billington SJ, Wright CL, Huggins AS, Katz ME, Rood JI. Delineation of the virulence-related locus (vrl) of Dichelobacter nodosus. Microbiology 1995; 141 ( Pt 9):2081-2089.

[11] Billington SJ, Huggins AS, Johanesen PA, et al. Complete nucleotide sequence of the 27-kilobase virulence related locus $(v r l)$ of $\mathrm{Di}$ chelobacter nodosus: evidence for extrachromosomal origin. Infect Immun 1999; 67(3):1277-1286.

[12] Rood JI, Howarth PA, Haring V, et al. Comparison of gene probe and conventional methods for the differentiation of ovine footrot isolates of Dichelobacter nodosus. Vet Microbiol 1996; 52(12):127-141.

[13] Cheetham BF, Tanjung LR, Sutherland M, et al. Improved diagnosis of virulent ovine footrot using the intA gene. Vet Microbiol 2006; 116:166-174.

[14] Whittle G, Bloomfield GA, Katz ME, Cheetham BF. The sitespecific integration of genetic elements may modulate thermostable protease production, a virulence factor in Dichelobacter nodosus, the causative agent of ovine footrot. Microbiology 1999; 145:28452855.

[15] Lawhon SD, Frye JG, Suyemoto M, Porwollik S, McClelland M, Altier C. Global regulation by CsrA in Salmonella typhimurium. Mol Microbiol 2003; 48(6):1633-1645.

[16] Barnard FM, Loughlin MF, Fainberg HP, et al. Global regulation of virulence and the stress response by CsrA in the highly adapted human gastric pathogen Helicobacter pylori. Mol Microbiol 2004; 51(1):15-32.

[17] Molofsky AB, Swanson MS. Legionella pneumophila CsrA is a pivotal repressor of transmission traits and activator of replication. Mol Microbiol 2003; 50(2):445-461.

[18] Mukherjee A, Cui Y, Liu Y, Dumenyo CK, Chatterjee AK. Global regulation in Erwinia species by Erwinia carotovora rsmA, a homologue of Escherichia coli csrA: repression of secondary metabolites, pathogenicity and hypersensitive reaction. Microbiology 1996; 142:427-434.

[19] Clements MO, Eriksson S, Thompson A, et al. Polynucleotide phosphorylase is a global regulator of virulence and persistency in 
Salmonella enterica. Proc Natl Acad Sci USA 2002; 99(13):87848789.

[20] Withey JH, Friedman DI. The biological roles of trans-translation. Curr Opin Microbiol 2002; 5(2):154-159.

[21] Julio SM, Heithoff DM, Mahan MJ. ssrA (tmRNA) plays a role in Salmonella enterica Serovar Typhimurium pathogenesis. J Bacteriol 2000; 182(6):1558-1563.

[22] Woods WH, Egan JB. Prophage induction of noninducible coliphage 186. J Virol 1974; 14(6):1349-1356.

[23] Horne RW. Negative staining methods. In: Kay DH, editor. Techniques for electron microscopy. Oxford: Blackwell Scientific Publications, 1965: 328-355.

[24] Hayat MA. Basic techniques for transmission electron microscopy. Orlando, FL: Academic Press, 1986.

[25] Ackermann HW, Audurier A, Berthiaume L, Jones LA, Mayo JA, Vidaver AK. Guidelines for bacteriophage characterization. Adv Virus Res 1978; 23:1-24.

[26] Murphy FA, Fauquet CM, Bishop DHL, et al. Virus taxonomy classification and nomenclature of viruses, sixth report of the international committee on the taxonomy of viruses. Archives of Virology, Supplement 10. New York, NY: Springer-Verlag, 1995.

[27] Ackerman H-W, Dubow MS. Viruses of prokaryotes. Boca Raton, FL: CRC Press, 1987.

[28] Stanley E, Fitzgerald GF, Le Marrec C, Fayard B, van Sinderen D. Sequence analysis and characterization of phi O1205, a temperate bacteriophage infecting Streptococcus thermophilus CNRZ1205. Microbiology 1997; 143:3417-3429.

[29] Pierson LS, Kahn ML. Integration of satellite bacteriophage P4 in Escherichia coli, DNA sequencing of the phage and host regions involved in site- specific recombination. J Mol Biol 1987; 196:487496.

[30] Kaneko T, Nakamura Y, Sato S, et al. Complete genome structure of the nitrogen-fixing symbiotic bacterium Mesorhizobium loti. DNA Res 2000; 7(6):331-338.

[31] Argos P, Landy A, Abremski K, et al. The integrase family of sitespecific recombinases: regional similarities and global diversity. EMBO J 1986; 5:433-440.
[32] Myers GS, Parker D, Al Hasani K, et al. Genome sequence and identification of candidate vaccine antigens from the animal pathogen Dichelobacter nodosus. Nat Biotechnol 2007; 25(5):569-575.

[33] Pierson LS, Kahn ML. Cloning of the integration and attachment regions of bacteriophage P4. Mol Gen Genet 1984; 195:44-51.

[34] Lindsey DF, Mullin DA, Walker JR. Characterization of the cryptic lambdoid prophage DLP12 of Escherichia coli and overlap of the DLP12 integrase with the tRNA gene $\arg U$. J Bacteriol 1989; 171:6197-6205.

[35] Naito T, Kusano K, Kobayashi I. Selfish behavior of restrictionmodification systems. Science 1995; 267(5199):897-899.

[36] Kondo Y, Toyoda A, Fukushi H, et al. Cloning and characterization of a pair of genes that stimulate the production and secretion of Zymomonas mobilis extracellular levansucrase and invertase. Biosci Biotechnol Biochem 1994; 58(3):526-530.

[37] Liu M, Gingery M, Doulatov SR, et al. Genomic and genetic analysis of Bordetella bacteriophages encoding reverse transcriptase-mediated tropism-switching cassettes. J Bacteriol 2004; 186(5):1503-1517.

[38] Bell KS, Sebaihia M, Pritchard L, et al. Genome sequence of the enterobacterial phytopathogen Erwinia carotovora subsp. atroseptica and characterization of virulence factors. Proc Natl Acad Sci USA 2004; 101(30):11105-11110.

[39] Alonso JC, Luder G, Stiege AC, Chai S, Weise F, Trautner TA. The complete nucleotide sequence and functional organization of Bacillus subtilis bacteriophage SPP1. Gene 1997; 204(1-2):201212.

[40] Catalano CE, Cue D, Feiss M. Virus DNA packaging: the strategy used by phage lambda. Mol Microbiol 1995; 16(6):1075-1086.

[41] Feiss M, Kobayashi I, Widner W. Separate sites for binding and nicking of bacteriophage lambda DNA by terminase. Proc Natl Acad Sci USA 1983; 80(4):955-959.

[42] Higgins RR, Becker A. Chromosome end formation in phage lambda, catalyzed by terminase, is controlled by two DNA elements of $\cos$, $\cos \mathrm{N}$ and R3, and by ATP. EMBO J 1994; 13(24):6152-6161.

[43] Deho G, Zangrossi S, Sabbattini P, Sironi G, Ghisotti D. Bacteriophage P4 immunity controlled by small RNAs via transcription termination. Mol Microbiol 1992; 6(22):3415-3425. 